\title{
Canadian Urological Association guideline: Muscle-invasive bladder cancer
}

Girish S. Kulkarni ${ }^{1}$; Peter C. Black ${ }^{2}$; Srikala S. Sridhar ${ }^{3}$; Anil Kapoor ${ }^{4}$; Alexandre R. Zlotta ${ }^{1}$; Bobby Shayegan ${ }^{4}$; Ricardo Rendon ${ }^{5}$; Peter Chung ${ }^{6}$; Theodorus van der Kwast ${ }^{7}$; Nimira Alimohamed $^{8}$; Yves Fradet $^{9}$; Wassim Kassouf ${ }^{10}$

${ }^{1}$ Division of Urology, Departments of Surgery and Surgical Oncology, Princess Margaret Cancer Centre, University Health Network, University of Toronto, Toronto, ON, Canada; ${ }^{2}$ Department of Urologic Sciences, University of British Columbia,Vancouver, BC, Canada; ${ }^{3}$ Division of Hematology and Medical Oncology, Princess Margaret Cancer Centre, University Health Network, University of Toronto, Toronto, ON, Canada; ${ }^{4}$ Section of Urology, Department of Surgery, McMaster University, Hamilton, ON, Canada; ${ }^{5}$ Department of Urology, Dalhousie University, Halifax, NS, Canada; ${ }^{6}$ Radiation Medicine Program, Princess Margaret Cancer Centre, University Health Network, Department of Radiation Oncology, University of Toronto, Toronto, ON, Canada; ${ }^{7}$ Laboratory Medicine Program, University Hospital Network, Toronto, ON, Canada; ${ }^{8}$ Division of Medical Oncology, Department of Oncology, University of Calgary, Calgary, AB, Canada; ${ }^{9}$ Division of Urology, Department of Surgery, Laval University, Quebec City, QC, Canada; ${ }^{10}$ Department of Urology, McGill University Health Centre, Montreal, QC, Canada

Cite as: Can Urol Assoc J 2019 January 31; Epub ahead of print. http://dx.doi.org/10.5489/cuaj.5902

Published online January 31, 2019

$* * *$

\section{Introduction}

On average, an estimated 9000 incident cases of bladder cancer are diagnosed in Canada annually. ${ }^{1}$ Of these, approximately $25 \%$ will be muscle-invasive at presentation. Muscle invasive bladder cancer (MIBC) possesses an aggressive biology that portends metastatic disease. Overall, the 5 year mortality of patients diagnosed with localized MIBC is approximately 40$50 \% .^{2,3}$ In the setting of metastatic disease, long-term survival is rare. To help streamline treatment and optimize care, the Canadian Urological Association commissioned the creation of a national guideline on MIBC.

\section{Methods}

All relevant articles on MIBC and metastatic bladder cancer were sought using a combination of Medline and EMBASE searches. The search strategy involved the following key words: "bladder cancer”, "urothelial carcinoma”, “invasive”, “muscle invasive” and “metastatic.” Filters included English language, human studies and an index date between 2000 and 2017, inclusive.

Bibliographies of review articles were searched for any missing articles not captured by our search strategy. Recently published guidelines from the European Association of Urology ${ }^{4}$, American Society of Clinical Oncology ${ }^{5}$, American Urological Association ${ }^{6}$ and the National Comprehensive Cancer Network ${ }^{7}$ were also considered for additional content. 
An expert panel of academic clinicians with experience managing patients with MIBC and metastatic bladder cancer was then gathered to facilitate guideline creation. Best practice statements were generated for broad categories of "Diagnosis”, “TURBT Pathology”, "Staging”, “Treatment”, “Supportive and Palliative Care”, "Follow Up and Quality of Life” and "Future Directions". Final guideline statements were determined by iterative feedback and consensus by the expert panel. A brief discussion for each category highlighting salient issues has been included as well.

Whenever possible, guideline statements have been assigned a level of evidence (LE) based on the Oxford Centre for Evidence-based Medicine (see Appendix). A subjective consensus recommendation ("Strong”, "Moderate" "Weak") based on the quantity, quality and consistency of the evidence available has also been applied to each statement. ",9 "Strong” recommendations have been given if high quality, consistent evidence supports the statement or for situations where wide consensus amongst experts is present and additional research is unlikely to modify confidence in the statement. A "weak" recommendation, on the contrary, represents the scenario where the level of evidence available is poor or where significant uncertainty in the guideline statement exists. Where recommendations for treatment were supported primarily by biologically plausible mechanisms without explicit data, a label of “Expert Opinion” was applied.

\section{Evidence synthesis: Guideline statements and discussion}

\section{Multidisciplinary initial assessment}

- MIBC patients should be assessed in a multidisciplinary manner whenever possible (LE 3, strong recommendation).

All patients with suspected MIBC require a thorough history and physical examination to determine bladder function, presence of comorbid disease and overall performance status. Cystoscopy should be included as part of the initial assessment as it provides an indication of tumour location, disease extent and is invaluable in the initial assessment of all bladder cancers. While traditional therapy in the localized MIBC setting has been radical cystectomy, contemporary care should involve a multi-disciplinary approach. ${ }^{10,11,12}$ Since many patients with MIBC suffer from significant comorbid disease that may influence subsequent multidisciplinary management decisions, most patients with MIBC would benefit from input at a Multidisciplinary Case Conference where eligibility for perioperative chemotherapy, radical surgery, and radiotherapy may be determined. All patients should also be considered for appropriate clinical trials. 


\section{Diagnosis}

- MIBC should be diagnosed with a good quality transurethral resection of the bladder tumour (TURBT) including muscularis propria that confirms muscle invasion (LE 3, strong recommendation).

The diagnosis of localized bladder cancer usually begins with a high quality TURBT. ${ }^{13}$ Where possible, clearance of all macroscopic disease is recommended to ensure optimal pathologic analysis and to render the patient clinically disease free such that all treatment options, including bladder preservation with trimodal therapy, are available to the patient. ${ }^{14}$ Despite this goal, it is recognized that complete transurethral resection for particularly large tumours may be unsafe and thus impossible.

In the majority of cases, inadequate sampling of the muscularis propria of the bladder precludes a MIBC diagnosis. In these cases, repeat resection should be strongly considered. However, in those rare instances where clear radiographic or clinical (e.g. bimanual examination) evidence supports a clear-cut clinical diagnosis of MIBC and where (i) tumour size precludes safely performing a complete TURBT and/or (ii) complete TURBT is simply not feasible, tumour tissue should still be procured to establish a bladder cancer diagnosis and determine final histology.

\section{TURBT pathology}

- The histological type (i.e. urothelial, squamous cell, small cell carcinoma, etc) of the tumour should be reported. For tumours displaying mixed histology, each histologic type present in the sample should be noted ( $L E 3$, strong recommendation).

- Additional pathological data including depth of invasion, grade, the presence of concomitant carcinoma-in-situ (CIS) and lymphovascular invasion (LVI) should be noted (LE 3, strong recommendation).

- Divergent differentiation of urothelial carcinoma (e.g. urothelial carcinoma with squamous, glandular or sarcomatoid differentiation), including variant histology (i.e. micropapillary, plasmacytoid, nested variant, etc.) should be recorded as well as an estimate of the proportion of variant histology (LE 3, strong recommendation).

- Pathology review by a second pathologist, preferably a dedicated genito-urinary pathologist, is recommended for all cases of variant histology (LE 3, moderate recommendation).

While urothelial carcinoma comprises $90 \%$ of MIBC, histologies such as adenocarcinoma, squamous cell carcinoma and rarer primary histologies warrant special consideration as these tumour types generally present at a more advanced stage and thus carry a higher risk of recurrence and worse overall prognosis. ${ }^{15,16,17}$ Other established negative prognostic factors which may influence subsequent treatment and surveillance decisions include the presence of LVI and CIS. Specifically, concomitant CIS has been linked to higher rates of recurrence after RC and worse cancer-specific survival in patients with pT2 or less disease at 
RC. ${ }^{18}$ It has also been associated with radioresistance ${ }^{19}$ Patients with LVI also have more aggressive disease and its documentation may reinforce the need for NAC. ${ }^{20,21}$ Variant urothelial histology (i.e. micropapillary, nested/large nested, plasmacytoid, sarcomatoid, microcystic, small tubules or lymphoepithelioma-type urothelial carcinoma) or extensive glandular/squamous differentiation also portend poorer outcomes and more advanced disease at presentation. Given reported evidence of significant inter-observer variability in pathologist's abilities to discern variant histology, all tumours displaying variant histology should undergo pathological re-review, preferably by an expert genito-urinary pathologist. ${ }^{22}$

\section{Staging}

- Examination under anesthesia should be performed immediately after TURBT to accurately determine clinical stage and resectability ( $L E 3$, moderate recommendation).

- Computed Tomography (CT) of the chest, abdomen and pelvis is the ideal treatment modality to stage localized MIBC and metastatic bladder cancer (LE 3, moderate recommendation).

- Magnetic resonance imaging (MRI) is an option to determine the local extent of disease (LE 3, moderate recommendation).

- Bone scans are not considered mandatory but should be obtained in the setting of an elevated ALP, hypercalcemia or bony pain (LE 3, moderate recommendation).

- Currently, the role for PET CT in the staging of bladder cancer remains undefined (LE 4, weak recommendation).

Prior to embarking on therapy, an accurate assessment of clinical stage is necessary. In addition to a thorough examination under anesthesia, axial imaging (CT or MRI) of the abdomen and pelvis to rule out nodal or metastatic disease is mandatory. These tests also aid in the determination of local extent of the disease, information that is required for surgical or radiation planning. Contrast-enhanced studies should be performed, where renal function allows, with delayed images (i.e. CT or MR urography) to assess for concomitant upper tract disease and to rule out hydronephrosis. Chest imaging (CT or X-ray) should also be performed to rule out metastatic disease or concomitant lung cancer given the preponderance of smoking in urothelial carcinoma patients, with CT of the chest providing the most sensitivity to detect metastases. Currently, there is insufficient evidence to recommend routine use of PET CT imaging in bladder cancer patients although guidelines from other organizations have suggested its incorporation in the staging and follow up of MIBC albeit with lower levels of evidence. ${ }^{7}$

\section{Treatment}

\section{Chemotherapy}

- All eligible patients with cT2-T4a NO MO urothelial carcinoma of the bladder should be encouraged to receive cisplatin-based combination chemotherapy (GC, MVAC or dd- 
MVAC) as neoadjuvant therapy (NAC) prior to radical local therapy (LE 1, strong recommendation).

- Absolute contraindications to NAC include Eastern Cooperative Group (ECOG) status of 2 or higher, grade 2 hearing loss or neuropathy, untreated infection, heart failure (NYHA Class III and IV) and an eGFR $\leq 50 \mathrm{ml} / \mathrm{min} / 1.73 \mathrm{~m}^{2}$. Relative contraindications for NAC include an eGFR between 50 and $60 \mathrm{ml} / \mathrm{min} / 1.73 \mathrm{~m}^{2}$, a history of recurrent infection and concomitant immunosuppression (LE 2, strong recommendation).

- Patients with contraindications to cisplatin-based neoadjuvant chemotherapy should proceed directly to radical local therapy (LE 2, strong recommendation).

- To optimize renal function in patients considering and/or eligible for NAC, malignant ureteric obstruction should be relieved via percutaneous drainage nephrostomy tubes (expert opinion).

- After 2/4 cycles of Gemcitabine/Cisplatin or conventional MVAC NAC, restaging should be performed to ensure treatment response or stable disease during chemotherapy. In the event of non-metastatic progressive disease or significant toxicity to chemotherapy that precludes its delivery, NAC should be discontinued and cystectomy performed within 4-6 weeks of last chemotherapy. Patients receiving ddMVAC, given every 2 weeks, do not need restaging in the midst of chemotherapy as the short course of treatment precludes the need for imaging (expert opinion).

- Patients receiving neoadjuvant chemotherapy should ideally undergo cystectomy 4-6 weeks after completion of NAC and at most within 10 weeks of the last dose of chemotherapy to avoid compromising survival ( $L E 3$, moderate recommendation).

- The role of neoadjuvant chemotherapy in pure non-urothelial carcinoma (squamous cell carcinoma, adenocarcinoma, etc.) is not defined and should not be utilized ( $L E 3$, strong recommendation).

- In patients who do not receive neoadjuvant chemotherapy prior to cystectomy, adjuvant cisplatin-based combination chemotherapy (GC, MVAC or dd-MVAC) should be offered to those eligible patients with pT3/T4 and/or $\mathbf{N}+$ disease (LE 2, strong recommendation).

- Patients with non-metastatic, clinically unresectable, cT4b or cN+tumours should be offered induction (primary) cisplatin-based combination chemotherapy if eligible or an alternative combination chemotherapy regimen if platinum-ineligible (e.g.

Gemcitabine/Carboplatin) or enrolment in a clinical trial, if available. Consolidative radical therapy should be considered after induction chemotherapy, where possible, particularly in those with responsive or stable disease (LE 3, weak recommendation).

Two large phase III clinical trials have demonstrated a mortality benefit with the use of neoadjuvant chemotherapy prior to local radical treatment. ${ }^{23,24}$ Meta-analyses combining individual patient data from these trials with numerous phase II trials have suggested an absolute survival benefit of $5 \%$ at 5 years (number-needed-to-treat of 20) and a 13\% mortality relative risk reduction in patients receiving NAC. ${ }^{25}$ It is important to note that evidence supportive of 
NAC is primarily derived in the urothelial carcinoma setting, with a lack of robust data supporting NAC in pure non-urothelial histologies. An exception to this rule is small cell carcinoma of the bladder where NAC serves as part of the mainstay of treatment. Despite level 1 evidence supporting NAC, uptake has been poor, with recent data still demonstrating only a $27 \%$ compliance rate in the modern era. ${ }^{26}$ Reasons posited for slow adoption include concerns regarding delayed definitive care, the risk of venous thromboembolism during NAC, NAC-related mortality and the non-selective nature of NAC. Countering these concerns is the randomized nature of the trials supporting NAC which, by definition, already account for chemotherapy-induced venous thromboembolism (which has a higher rate of occurrence in the NAC population, risk ratio $=3.39$, 95\% CI: $1.39-8.24)^{27}$, death directly attributable to chemotherapy and any possible delays in radical therapy from NAC. ${ }^{28}$ Even with the aforementioned potential shortcomings, the NAC meta-analysis nevertheless reported a survival benefit. A number of reports also suggest that NAC does not increase perioperative morbidity or complication rates lending further support to its usage. ${ }^{29,30}$ Ample time (usually 2-3 weeks) for recovery of complete blood count parameters and optimization of patient fitness after completion of NAC is required prior to delivery of definitive radical therapy. The ideal time for radical therapy after NAC, as adopted by the Canadian Association of Genitourinary Medical Oncologists (CAGMO), is within 4-6 weeks although a maximal window of 10 weeks has not been shown to compromise outcomes. ${ }^{31,32}$

To date, there are no randomized trials comparing NAC to adjuvant chemotherapy (AC). While data do support use of adjuvant chemotherapy (AC), with an approximate 23\% survival benefit (HR 0.77, 95\% CI 0.59- 0.99) based on meta-analyzed data ${ }^{33}$, no single phase III trial has demonstrated an overall survival benefit with AC compared to observation. Even the most recent Phase III EORTC trial in this setting, while demonstrating a significant progression-free survival benefit (HR 0.54; 95\% CI 0.40-0.73), was ultimately underpowered to demonstrate an overall survival benefit (HR 0.78; 95\% CI 0.56-1.08). ${ }^{34}$ The overall quality of evidence also favours NAC as the perioperative chemotherapy option of choice as many AC trials suffered from poor accrual, early termination and lack of power. Furthermore, many patients after radical cystectomy experience renal function deterioration, resulting in an estimated $24-52 \%$ rate of ineligibility to receive AC postoperatively depending on the criteria used. ${ }^{35}$ Postoperative complications may also limit AC use, excluding approximately $30 \%$ of patients who may have been eligible from receiving necessary treatment ${ }^{36}$ Given that metastatic disease is the most likely cause of death in patients with MIBC, an approach that maximizes the ability to administer multi-modal therapy should be adopted, thus favouring a NAC approach. 
Surgical management

- The standard therapy for localized MIBC is radical cystectomy ( $L E$ 1, strong recommendation).

- $\quad$ The optimal timing of radical cystectomy where NAC has not been administered is within 6 weeks of TURBT (LE 3, moderate recommendation).

- Patients scheduled for radical cystectomy are recommended to receive perioperative optimization according to endorsed Enhanced Recovery after Abdominal Surgery (ERAS) protocols (LE 2, moderate recommendation).

- In male patients, radical cystectomy entails removal of the bladder and prostate enbloc. A nerve-sparing procedure can be safely offered to select patients interested in preserving sexual function ( $L E 3$, moderate recommendation).

- In female patients, radical cystectomy entails removal of the bladder, reproductive organs (uterus and ovaries) and the anterior vagina. In situations where the tumour location allows (i.e. anterior tumours), a female organ sparing (i.e. uterus, ovaries and/or vagina) operation can be offered to women interested in preserving sexual and/or reproductive function ( $L E$ 3, moderate recommendation).

- Bilateral pelvic lymph node dissection with removal, at minimum, of the obturator, external iliac and internal iliac lymph nodes should be performed in all patients ( $L E 3$, strong recommendation).

- Orthotopic urinary diversion should be offered to all eligible patients as an alternative to an ileal conduit. An intraoperative frozen section evaluation of the urethral margin should be performed prior to creating an orthotopic diversion (LE 3, moderate recommendation).

- Urethrectomy should be performed in men with high grade or invasive urethral disease distal to the prostatic urethra, a positive urethral margin or suspected prostatic stromal involvement in men and bladder neck tumours in women (LE 3, moderate recommendation).

- Both laparoscopic/robotic and open approaches are acceptable methods to perform radical cystectomy with comparable cancer outcomes ( $L E 1$, strong recommendation).

- Partial cystectomy for MIBC is discouraged and should only be considered in specific situations: unifocal, small tumour $<2 \mathrm{~cm}$, dome location, good bladder capacity, no hydronephrosis, minimal to no concomitant CIS. Random bladder biopsies (plus prostatic urethral biopsy) should be performed prior to partial cystectomy to rule out occult disease. Pelvic lymph node dissection should be performed at the time of partial cystectomy (LE 3, moderate recommendation).

Radical cystectomy is the standard surgical therapy for MIBC with established, long term oncologic outcomes. ${ }^{37}$ Perioperative patient counselling regarding the extent of the operation and the associated gender-specific organs to be resected should be documented preoperatively. At a minimum, a standard pelvic lymph node dissection constituting the external iliac, obturator and internal iliac lymph nodes should be removed for therapeutic and staging purposes. ${ }^{38,39,40,41,42}$ 
Many retrospective studies have suggested a survival benefit of extending the cystectomy lymph node dissection boundaries to a level as high as the inferior mesenteric artery ${ }^{43,44}$,and RCT data that may definitively support this observation is pending maturity. ${ }^{45}$

Treatment of MIBC can lead to short term and long term side effects that should be discussed with patients. In one of the largest series reporting on 90 day complications after cystectomy, approximately 64\% of 1142 patients experienced one or more complications with $83 \%$ of those deemed significant (Clavien 2-5 on the Clavien-Dindo Classification system). ${ }^{38}$ Given the burden of treatment of radical cystectomy, detailed perioperative planning should be undertaken to optimize outcomes. Excessive delays from TURBT to cystectomy should be avoided in patients not receiving NAC. ${ }^{46}$ Eligibility for continent diversion should be determined for all patients and final decisions on the type of diversion should be made based on renal and hepatic function, comorbidity/performance status, patient preference and tumour location, with the latter also guiding discussions regarding concomitant urethrectomy at the time of cystectomy. Growing data support Enhanced Recovery after Abdominal Surgery (ERAS) protocols as a means of decreasing length of stay and postoperative complications with radical cystectomy. ${ }^{47,48}$ Specifically, a Canadian Urological Association Continuing Professional Development slide deck on the topic has been created to guide urologists' implementation of ERAS for cystectomy. $^{49}$

Cystectomy pathology

- The final pathology report should contain the following elements: histology (including variants), stage, grade, presence of concomitant CIS, presence of LVI, number of lymph nodes, number of positive lymph nodes, and surgical margin status ( $L E 3$, strong recommendation).

- Assessment of accompanying reproductive organs (prostate, uterus, cervix, ovaries, vagina) should be performed to rule out occult secondary malignancy and for determination of final pathologic stage (LE 3, moderate recommendation).

An accurate assessment of pathologic stage in cystectomy specimens is of utmost importance. Synoptic reporting of pathologic data is encouraged to standardize nomenclature across institutions. Pathology data generally guide discussions about prognosis with worse outcomes expected with higher stage disease or with concomitant CIS or LVI. ${ }^{18,50}$ Receipt of adjuvant chemotherapy is also dependent on accurate pathologic assessment and is generally recommended in patients with node positive and/or pT3/4 disease. ${ }^{36}$ Synoptic pathology reporting also provides data that can serve as surgical quality indicators (e.g. margin status, number of nodes removed, dissection template, etc.). 
Radiotherapy

- Trimodal therapy (TMT; radical TURBT + external beam radiotherapy + concomitant chemotherapy) can be offered to select patients wishing to preserve their bladder, those unfit for cystectomy or those refusing cystectomy (LE 3, moderate recommendation).

- Ideal tumour and patient characteristics for TMT are as follows: small $(<5 \mathrm{~cm})$, unifocal, no CIS, no hydronephrosis, good bladder function, patient motivated for bladder preservation ( $L E 3$, moderate recommendation).

- With TMT, maximal/radical TURBT should be performed to clear all visible tumour prior to initiation of chemoradiation (LE 3, moderate recommendation).

- Radiotherapy as monotherapy in the treatment of localized MIBC is only acceptable in patients who are ineligible for both radical cystectomy and chemotherapy. Otherwise radiation should be offered in combination with either cisplatin or 5-FU/MMC chemotherapy (LE 1, strong recommendation) or Gemcitabine (LE 2, strong recommendation)

- Currently, there is no well-defined role for neoadjuvant or adjuvant radiotherapy in the setting of localized MIBC (LE 3, moderate recommendation).

Although radical cystectomy is the de facto gold standard surgical therapy for MIBC, TMT can also be offered to select patients who seek bladder preservation. Optimal patient selection is key in this setting with criteria as delineated above and provision of care in a multidisciplinary setting likely yielding the most robust outcomes. A recent report demonstrated that, in carefully selected patients, TMT offered in a multidisciplinary bladder cancer clinic yielded moderate-term disease-specific survival rates rivalling that of radical cystectomy (73\% for RC, $77 \%$ for TMT). ${ }^{10}$ Ultimately, only approximately $20-25 \%$ of surgically fit patients will meet the criteria for TMT bladder preservation.

In addition to TMT providing the opportunity for bladder preservation in select healthy, surgically fit patients, TMT offers the ability to extend treatment to patients who would otherwise go untreated. Population-based data demonstrate that many patients are poor surgical candidates thus leading to undertreatment of non-metastatic MIBC in approximately $50 \%$ of patients. ${ }^{51}$ Trimodal therapy can safely be offered to patients unfit for surgical therapy thus providing some benefit for these patients. ${ }^{52}$

Where possible, radiation should be administered with radio-sensitizing chemotherapy. Radiotherapy alone as monotherapy has been shown in a large randomized control trial to be inferior to radiotherapy plus chemotherapy. ${ }^{53}$ A smaller randomized NRG/RTOG trial demonstrated similar 3-year distant metastases-free survival regardless if the chemotherapeutic regimen utilized was 5-FU-based or gemcitabine-based. ${ }^{54}$ 
Unresectable and oligometastatic disease

- First line therapy for unresectable urothelial carcinoma is cisplatin-based combination chemotherapy with either GC, MVAC or ddMVAC. In cisplatin-ineligible patients (i.e. absolute contraindications to cisplatin; see section on NAC above), carboplatin combination chemotherapy or single agent chemotherapy may be substituted as first line therapy (LE 1, strong recommendation).

- At present, there is insufficient data to support a role for resection of oligometastatic disease as part of primary therapy (synchronous) or as management of recurrent disease (metachronous) (expert opinion).

- Consideration of consolidative surgery (radical cystectomy) combined with oligometastectomy in initially unresectable patients experiencing a significant $C R$ or $P R$ must be on a case-by-case basis after multidisciplinary tumour board discussion (LE 4, weak recommendation).

Patients with unresectable or oligometastatic disease at presentation should undergo primary chemotherapy. In those with an excellent response (CR or PR), multidisciplinary cancer conference discussion regarding the role of consolidative therapy of the primary and metastatic lesion(s) should be undertaken. No randomized data support oligometastectomy in the synchronous setting (i.e. radical cystectomy plus resection of oligometastatic sites in one setting), yet retrospective data suggest that carefully selected patients who undergo resection of the primary along with limited metasatectomy can achieve durable long term survival rates of $10-20 \% .{ }^{55,56}$ Oligometastatic sites that have been resected include most commonly the retroperitoneal lymph nodes, lung metastases and bone. ${ }^{57}$ Factors to be considered prior to embarking on such extensive surgical consolidation include: i) response to primary chemotherapy, ii) extent of disease, iii) feasibility of resection, iv) performance status and v) patient motivation.

Followup and quality of life

- Quality of Life (QOL) in the form of a validated Patient Reported Outcome (PRO) measure or QOL instrument should be captured for all patients at each visit ( $L E 3$, weak recommendation).

- Followup schedules should be tailored to final pathologic TNM staging (LE 3, weak recommendation).

- Followup visits after radical cystectomy should include a metastatic survey including an investigation for upper tract recurrence, assessment for hydronephrosis and laboratory studies to detect metabolic complications of diversion ( $L E 3$, moderate recommendation).

- In patients at high risk for urethral or upper tract recurrence, urethral washings +/urethroscopy and urine should be collected for cytologic examination at interval follow up visits (LE 3, moderate recommendation). 
- Patients treated with a bladder preservation approach (radiotherapy-based or partial cystectomy) should also receive, in addition to the same investigations performed for radical cystectomy patients, long term cystoscopic evaluation at each follow up visit to survey the remaining urothelium ( $L E 3$, strong recommendation).

- Intravesical recurrences after bladder preservation may be managed as per primary bladder tumours based on pathologic assessment after TURBT (LE 3, weak recommendation). Careful consideration for radical cystectomy should occur for high risk recurrences.

- Endoscopic biopsy is recommended following TMT to assess response (LE 3, moderate recommendation).

There are emerging data in the oncology literature supporting the need for quality of life and patient reported outcome assessment in cancer patients, with a recent study suggesting improved survival in patients reporting PROs. ${ }^{58}$ Bladder cancer is known to have a significant impact on QOL ${ }^{59,60}$ Cystectomy patients are at risk of long term sexual dysfunction, urinary complications (recurrent infections, uretero-enteric anastomotic strictures, stones, renal failure) and bowel dysfunction (diarrhea or constipation). ${ }^{61,62,63}$ TMT patients may experience sexual dysfunction, voiding and storage symptoms from urethral strictures or radiation cystitis, or bowel toxicity (radiation enteritis or proctitis). ${ }^{64,65}$ Downstream toxicity from perioperative chemotherapy may also occur (e.g. coronary artery disease, peripheral neuropathy, ototoxicity). ${ }^{66}$

In addition to monitoring for recurrent disease, surveillance regimens should incorporate testing to detect such long term complications. While no randomized data support a single surveillance protocol, a risk-adapted approach based on tumour stage (risk of urothelial recurrence) and comorbidity status (competing risk of death) may better tailor follow up to maximize recurrence detection while minimizing the burden of surveillance ${ }^{67}$ Currently, EUA and NCCN guidelines recommend 5 years of risk-adapted by stage and 2 years of non-riskadapted surveillance, respectively. ${ }^{4,7}$ The Canadian Bladder Cancer Network recommends a stage-based strategy to detect recurrences and delineates a stage-specific surveillance protocol. ${ }^{68}$ Regardless of the follow up regimen chosen, it should ideally be incorporated into a MIBC patient survivorship program.

Supportive and palliative care

- For patients with localized, non-metastatic MIBC who are unfit for radical intervention (radical cystectomy or TMT), an aggressive endoscopic approach ("radical TURBT") can be performed to achieve local control (LE 3, weak recommendation).

- Palliative care consultation should be requested early on in the care of incurable/unresectable patients (LE 1, strong recommendation).

- Palliative cystectomy can be performed in select cases, with non-curative intent, for intractable hematuria or pelvic pain secondary to the bladder tumour ( $L E 3$, weak recommendation). 
- Palliative radiotherapy may also be offered for intractable hematuria or bony pain secondary to metastatic disease (LE 3, moderate recommendation).

- Palliative chemotherapy (e.g., gemcitabine) may be offered to patients with unresectable or metastatic disease who are ineligible for or have failed platinum-based combination chemotherapy (LE 3, moderate recommendation).

Patients with unresectable or metastatic disease should be offered an early palliative care referral as a number of oncology randomized controlled trials have demonstrated improvements in health-related quality of life and symptom control with prompt referral. ${ }^{69}$ Local options for patients with intact bladders include palliative TURBT for hematuria, palliative cystectomy or radiotherapy for intractable hematuria or pelvic pain and localized palliative radiotherapy for painful metastatic lesions. Systemic options include palliative chemotherapy with agents with minimal toxicity (e.g. gemcitabine monotherapy) or enrollment in a clinical trial if available. ${ }^{70,71}$

\section{Conclusion}

Muscle invasive bladder cancer is a potentially lethal malignancy that requires intensive, multidisciplinary care to maximize cure while minimizing the burden and toxicity of treatment. These guidelines establish a Canadian perspective on the management of this difficult disease.

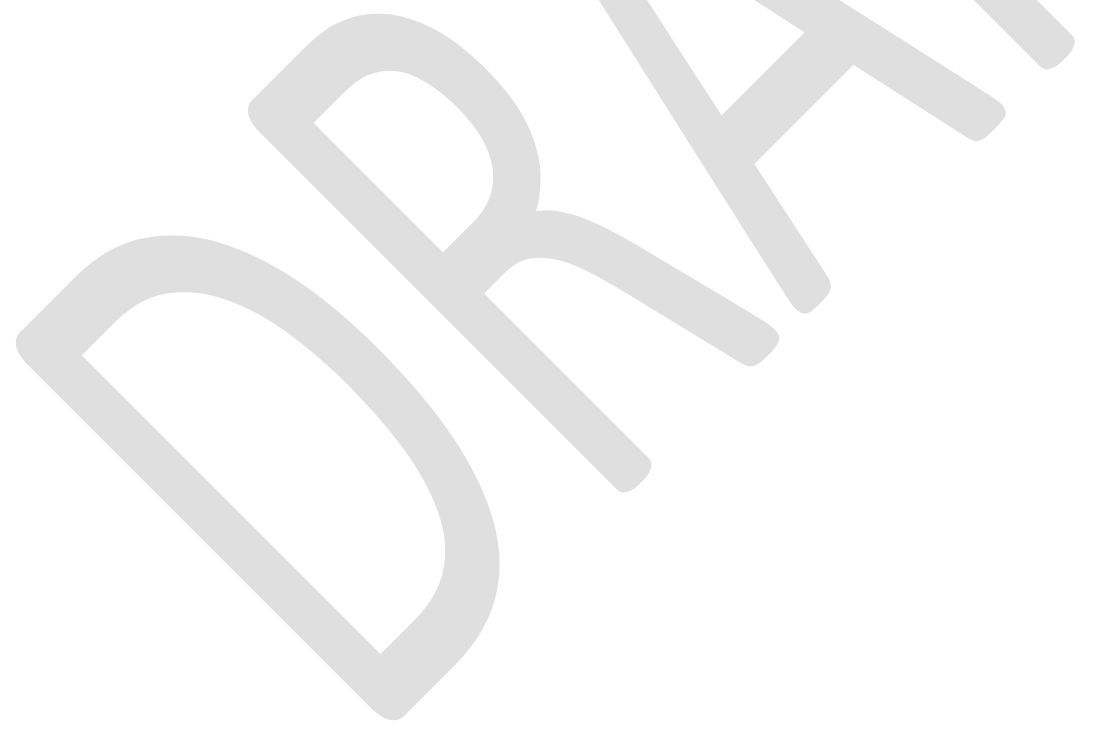




\section{References}

${ }^{1}$ Canadian Cancer Society’s Advisory Committee on Cancer Statistics. Canadian Cancer Statistics 2017. Toronto, ON: Canadian Cancer Society; 2017.

${ }^{2}$ Kulkarni GS, Urbach DR, Austin PC, et al. Higher surgeon and hospital volume improves longterm survival after radical cystectomy. Cancer 2013; 119:3546-54.

${ }^{3}$ Zakaria AS, Santos F, Dragomir A et al. Postoperative mortality and complications after radical cystectomy for bladder cancer in Quebec: A population-based analysis during the years 20002009.Can Urol Assoc J 2014; 8:259-67.

${ }^{4}$ Alfred Witjes J, Lebret T, Compérat EM, et al. Updated 2016 EAU Guidelines on muscleinvasive and metastatic bladder cancer. Eur Urol 2017; 71:462-75.

${ }^{5}$ Milowsky MI, Rumble RB, Booth CM, et al. Guideline on muscle-invasive and metastatic bladder cancer (European Association of Urology guideline): American Society of Clinical Oncology clinical practice guideline endorsement. J Clin Oncol 2016; 34:1945-52.

${ }^{6}$ Chang SS, Bochner BH, Chou RJ, et al. Treatment of non-metastatic muscle-invasive bladder cancer: AUA/ASCO/ASTRO/SUO guideline. Urol 2017; 198:552-59.

${ }^{7}$ NCCN Clinical Practice Guidelines in Oncology (NCCN Guideline): Bladder Cancer Version 2.2018 - February 7, 2018. www.nccn.org. Accessed February 8, 2018.

${ }^{8}$ The Grading of Recommendations Assessment, Development and Evaluation (GRADE) working group. 2018. http://www.gradeworkinggroup.org. Accessed Feb 1, 2018.

${ }^{9}$ Centre for Evidence-Based Medicine (CEBM). 2018. https://www.cebm.net. Accessed February 1, 2018.

${ }^{10}$ Kulkarni GS, Hermanns T, Wei Y, et al. Propensity Score Analysis of Radical Cystectomy Versus Bladder-Sparing Trimodal Therapy in the Setting of a Multidisciplinary Bladder Cancer Clinic. J Clin Oncol 2017; 35:2299-305.

${ }^{11}$ Chedgy EC and Black PC . Radical Cystectomy and the Multidisciplinary Management of Muscle-Invasive Bladder Cancer. JAMA Oncol 2016; 2:855-6.

12 Nayan M, Bhindi B, Yu JL, et al. The initiation of a multidisciplinary bladder cancer clinic and the uptake of neoadjuvant chemotherapy: A time-series analysis. Can Urol Assoc J 2016; 10:25-30.

13 James AC, Lee FC, Izard JP, et al. Role of maximal endoscopic resection before cystectomy for invasive urothelial bladder cancer. Clin Genitourin Cancer 2014; 12:287-91.

${ }^{14}$ Efstathiou JA, Spiegel DY, Shipley WU, et al. Long-term outcomes of selective bladder preservation by combined-modality therapy for invasive bladder cancer: the MGH experience. Eur Urol 2012; 61:705-11.

${ }^{15}$ Moschini M, Shariat SF, Lucianò R, et al. Pure but Not Mixed Histologic Variants Are Associated With Poor Survival at Radical Cystectomy in Bladder Cancer Patients. Clin Genitourin Cancer 2017; 15:e603-e607. doi: 10.1016/j.clgc.2016.12.006. Epub 2016 Dec 14. ${ }^{16}$ Dutta R, Abdelhalim A, Martin JW, et al. Effect of tumor location on survival in urinary bladder adenocarcinoma: A population-based analysis. Urol Oncol 2016; 34:531.e1-531.e6. doi: 10.1016/j.urolonc.2016.06.009.

${ }^{17}$ Chen C, Hu L, Chen Y, et al. The prognostic value of histological subtype in patients with metastatic bladder cancer. Oncotarget 2017; 8:28408-17. 
${ }^{18}$ Moschini M, Shariat SF, Abufaraj M, et al. The presence of carcinoma in situ at radical cystectomy increases the risk of urothelial recurrence: Implications for follow-up schemes. Urol Oncol 2017; 35:151.e17-151.e23. doi: 10.1016/j.urolonc.2016.11.003.

${ }^{19}$ Ploussard G, Daneshmand S, Efstathiou JA, et al. Critical analysis of bladder sparing with trimodal therapy in muscle-invasive bladder cancer: a systematic review. Eur Urol 2014; 66:12037.

${ }^{20}$ Streeper NM, Simons CM, Konety BR, et al. The significance of lymphovascular invasion in transurethral resection of bladder tumour and cystectomy specimens on the survival of patients with urothelial bladder cancer. BJU Int 2009; 103:475-9.

${ }^{21}$ Culp SH, Dickstein RJ, Grossman HB, et al. Refining patient selection for neoadjuvant chemotherapy before radical cystectomy. J Urol 2014; 191:40-7.

${ }^{22}$ Sangoi AR, Beck AH, Amin MB, et al. Interobserver reproducibility in the diagnosis of invasive micropapillary carcinoma of the urinary tract among urologic pathologists. Am J Surg Pathol 2010; 34:1367-76.

${ }^{23}$ International Collaboration of Trialists. International phase III trial assessing neoadjuvant cisplatin, methotrexate, and vinblastine chemotherapy for muscle-invasive bladder cancer: longterm results of the BA06 30894 trial. J Clin Oncol 2011; 29:2171-7.

${ }^{24}$ Grossman HB, Natale RB, Tangen CM, et al. Neoadjuvant chemotherapy plus cystectomy compared with cystectomy alone for locally advanced bladder cancer. $N$ Engl J Med 2003; 349:859-66.

${ }^{25}$ Advanced Bladder Cancer Meta-analysis Collaboration. Neoadjuvant chemotherapy in invasive bladder cancer: a systematic review and meta-analysis. Lancet 2003; 361:1927-34.

${ }^{26}$ Booth CM, Karim S, Brennan K, et al. Perioperative chemotherapy for bladder cancer in the general population: Are practice patterns finally changing? Urol Oncol 2017; pii: S10781439(17)30603-8. doi: 10.1016/j.urolonc.2017.11.015. [Epub ahead of print]

27 Zareba P, Patterson L, Pandya R, et al. Thromboembolic events in patients with urothelial carcinoma undergoing neoadjuvant chemotherapy and radical cystectomy. Urol Oncol 2014; 32:975-80.

${ }^{28}$ Dash A, Pettus JA 4th, Herr HW, et al. A role for neoadjuvant gemcitabine plus cisplatin in muscle-invasive urothelial carcinoma of the bladder: a retrospective experience. Cancer.2008; 113:2471-7.

${ }^{29}$ Johnson DC, Nielsen ME, Matthews J, et al. Neoadjuvant chemotherapy for bladder cancer does not increase risk of perioperative morbidity. BJU Int 2014; 114:221-8.

${ }^{30}$ Gandaglia G, Popa I, Abdollah F, et al. The effect of neoadjuvant chemotherapy on perioperative outcomes in patients who have bladder cancer treated with radical cystectomy: a population-based study. Eur Urol 2014; 66: 561-8.

${ }^{31}$ Seah JA, Blais N, North S, et al. Neoadjuvant chemotherapy should be administered to fit patients with newly diagnosed, potentially resectable muscle-invasive urothelial cancer of the bladder (MIBC): A 2013 CAGMO Consensus Statement and Call for a Streamlined Referral Process. Can Urol Assoc J 2013; 7:312-8.

${ }^{32}$ Alva AS, Tallman CT, He C, et al. Efficient delivery of radical cystectomy after neoadjuvant chemotherapy for muscle-invasive bladder cancer: a multidisciplinary approach. Cancer 2012; 118:44-53. 
${ }^{33}$ Leow JJ, Martin-Doyle W, Rajagopal PS, et al. Adjuvant chemotherapy for invasive bladder cancer: a 2013 updated systematic review and meta-analysis of randomized trials. Eur Urol 2014; 66:42-54.

${ }^{34}$ Sternberg CN, Skoneczna I, Kerst JM, et al. Immediate versus deferred chemotherapy after radical cystectomy in patients with pT3-pT4 or $\mathrm{N}+\mathrm{M} 0$ urothelial carcinoma of the bladder (EORTC 30994): an intergroup, open-label, randomised phase 3 trial. Lancet Oncol 2015; 16:7686.

${ }^{35}$ Dash A, Galsky MD, Vickers AJ, et al. Impact of renal impairment on eligibility for adjuvant cisplatin-based chemotherapy in patients with urothelial carcinoma of the bladder. Cancer 2006; 107:506-13.

${ }^{36}$ Donat SM, Shabsigh A, Savage C, et al. Potential impact of postoperative early complications on the timing of adjuvant chemotherapy in patients undergoing radical cystectomy: a highvolume tertiary cancer center experience. Eur Urol 2009; 55:177-85.

${ }^{37}$ Stein JP, Lieskovsky G, Cote R, et al.Radical cystectomy in the treatment of invasive bladder cancer: long-term results in 1,054 patients. J Clin Oncol 2001; 19:666-75.

${ }^{38}$ Steven K and Poulsen AL. Radical cystectomy and extended pelvic lymphadenectomy: survival of patients with lymph node metastasis above the bifurcation of the common iliac vessels treated with surgery only. J Urol 2007; 178:1218-23.

${ }^{39}$ Sundi D, Svatek RS, Nielsen ME, et al. Extent of pelvic lymph node dissection during radical cystectomy: is bigger better? Rev Urol 2014; 16:159-66.

${ }^{40}$ Larcher A, Sun M, Schiffmann J, et al. Differential effect on survival of pelvic lymph node dissection at radical cystectomy for muscle invasive bladder cancer. Eur J Surg Oncol 2015; 41:353-60.

${ }^{41}$ Abdi H, Pourmalek F, Gleave ME, et al. Balancing risk and benefit of extended pelvic lymph node dissection in patients undergoing radical cystectomy. World J Urol 2016; 34:41-8.

${ }^{42}$ Ho PL, Willis DL, Patil J, et al. Outcome of patients with clinically node-positive bladder cancer undergoing consolidative surgery after preoperative chemotherapy: The M.D. Anderson Cancer Center Experience. Urol Oncol 2016; 34:59.e1-8. doi: 10.1016/j.urolonc.2015.08.012. ${ }^{43}$ Wright JL, Lin DW and Porter MP. The association between extent of lymphadenectomy and survival among patients with lymph node metastases undergoing radical cystectomy. Cancer 2008; 112:2401-8.

${ }^{44}$ Wiesner C, Salzer A, Thomas C, et al. Cancer-specific survival after radical cystectomy and standardized extended lymphadenectomy for node-positive bladder cancer: prediction by lymph node positivity and density. BJU Int 2009; 104:331-5.

${ }^{45}$ Gschwend JE, Heck MM, Lehmann J et al. Extended Versus Limited Lymph Node Dissection in Bladder Cancer Patients Undergoing Radical Cystectomy: Survival Results from a Prospective, Randomized Trial. Eur Urol 2018; pii: S0302-2838(18)30737-1.

${ }^{46}$ Kulkarni GS, Urbach DR, Austin PC, et al. Longer wait times increase overall mortality in patients with bladder cancer. J Urol 2009; 182:1318-24.

${ }^{47}$ Tyson MD and Chang SS. Enhanced Recovery Pathways Versus Standard Care After Cystectomy: A Meta-analysis of the Effect on Perioperative Outcomes. Eur Urol 2016; 70:9951003. 
${ }^{48}$ Melnyk M, Casey RG, Black P, et al. Enhanced recovery after surgery (ERAS) protocols: Time to change practice? Can Urol Assoc J 2011; 5:342-8.

${ }^{49}$ Enhanced Recovery after Abdominal Surgery (ERAS) Protocols in the Urologic Setting: Focus on Radical Cystectomy. Canadian Urological Association. 2016. https://www.cua.org/en/cpdcme. Accessed Feb 1, 2018.

${ }^{50}$ Muppa P, Gupta S, Frank I, et al. Prognostic significance of lymphatic, vascular and perineural invasion for bladder cancer patients treated by radical cystectomy. Pathology. 2017; 49:259-266.

${ }^{51}$ Gore JL, Litwin MS, Lai J, et al. Urologic Diseases in America Project. Use of radical cystectomy for patients with invasive bladder cancer. J Natl Cancer Inst 2010; 102:802-11. ${ }^{52}$ Caffo O, Veccia A, Fellin G, et al. Trimodality treatment in the conservative management of infiltrating bladder cancer: a critical review of the literature. Crit Rev Oncol Hematol 2013; 86:176-90.

53 James ND, Hussain SA, Hall E, et al. BC2001 Investigators. Radiotherapy with or without chemotherapy in muscle-invasive bladder cancer. N Engl J Med 2012; 366:1477-88.

${ }^{54}$ Coen JJ, Zhang P, Saylor PJ, et al. Bladder Preservation With Twice-a-Day Radiation Plus Fluorouracil/Cisplatin or Once Daily Radiation Plus Gemcitabine for Muscle-Invasive Bladder Cancer: NRG/RTOG 0712-A Randomized Phase II Trial. J Clin Oncol 2018; Nov 15:JCO1800537.

${ }^{55}$ Ghadjar P, Burkhard FC, Gautschi O, et al. Induction chemotherapy for unresectable urothelial carcinoma of the bladder. BJU Int 2011; 107: 894-7.

${ }^{56}$ Yafi FA and Kassouf W. Management of patients with advanced bladder cancer following major response to systemic chemotherapy. Expert Rev Anticancer Ther 2009; 9:1757-64.

${ }^{57}$ Abe T, Matsumoto R and Shinohara N. Role of surgical consolidation in metastatic urothelial carcinoma. Curr Opin Urol 2016; 26:573-80.

${ }^{58}$ Basch E, Deal AM, Dueck AC, et al. Overall Survival Results of a Trial Assessing PatientReported Outcomes for Symptom Monitoring During Routine Cancer Treatment. JAMA. 2017; 318:197-198.

${ }^{59}$ Garg T, Connors JN, Ladd IG, et al. Defining Priorities to Improve Patient Experience in NonMuscle Invasive Bladder Cancer. Bladder Cancer 2018 ;4:121-128.

${ }^{60}$ Smith AB, Jaeger B, Pinheiro LC, et al. Impact of bladder cancer on health-related quality of life. BJU Int 2017; doi: 10.1111/bju.14047. [Epub ahead of print]

${ }^{61}$ Gilbert SM, Lai J, Saigal CS, et al. Urologic Diseases in America Project. Downstream complications following urinary diversion. Urol 2013; 190:916-22.

${ }^{62}$ Nazmy M, Yuh B, Kawachi M, et al. Early and late complications of robot-assisted radical cystectomy: a standardized analysis by urinary diversion type. J Urol 2014; 191:681-7.

${ }^{63}$ Shah SH, Movassaghi K, Skinner D, et al. Ureteroenteric Strictures After Open Radical Cystectomy and Urinary Diversion: The University of Southern California Experience. Urology 2015; 86:87-91.

${ }^{64}$ Huddart RA, Birtle A, Maynard L, et al. Clinical and patient-reported outcomes of SPARE - a randomised feasibility study of selective bladder preservation versus radical cystectomy. BJU Int 2017; 120:639-650.

${ }^{65}$ Feuerstein MA and Goenka A. Quality of Life Outcomes for Bladder Cancer Patients Undergoing Bladder Preservation with Radiotherapy. Curr Urol Rep 2015; 16:75. doi: 
10.1007/s11934-015-0547-1.

${ }^{66}$ Boer H, Proost JH, Nuver J, et al. Long-term exposure to circulating platinum is associated with late effects of treatment in testicular cancer survivors. Ann Oncol 2015; 26:2305-10.

${ }^{67}$ Stewart-Merrill SB, Boorjian SA, Thompson RH, et al. Evaluation of current surveillance guidelines following radical cystectomy and proposal of a novel risk-based approach. Urol Oncol 2015; 33:339.e1-8. doi: 10.1016/j.urolonc.2015.04.017. Epub 2015 May 29.

${ }^{68}$ Yafi FA, Aprikian AG, Fradet Y, et al. Surveillance guidelines based on recurrence patterns after radical cystectomy for bladder cancer: the Canadian Bladder Cancer Network experience. BJU Int 2012; 110:1327-24.

${ }^{69}$ Haun MW, Estel S, Rücker G, et al. Early palliative care for adults with advanced cancer. Cochrane Database Syst Rev 2017; 6:CD011129. doi: 10.1002/14651858.CD011129.pub2.

${ }^{70}$ Yafi FA, North S and Kassouf W. First- and second-line therapy for metastatic urothelial carcinoma of the bladder. Curr Oncol 2011; 18(1):e25-34.

${ }^{71}$ Vishnu P, Mathew J and Tan WW. Current therapeutic strategies for invasive and metastatic bladder cancer. Onco Targets Ther. 2011; 4:97-113. 
Figures and Tables

\begin{tabular}{|c|c|c|c|c|c|}
\hline Question & Level 1 & Level 2 & Level 3 & Level 4 & Level 5 \\
\hline Diagnosis & $\begin{array}{l}\text { SR of cross } \\
\text { sectional } \\
\text { studies with } \\
\text { consistently } \\
\text { applied } \\
\text { reference } \\
\text { standard and } \\
\text { blinding }\end{array}$ & $\begin{array}{c}\text { Individual } \\
\text { cross sectional } \\
\text { study with } \\
\text { consistently } \\
\text { applied } \\
\text { reference } \\
\text { standard and } \\
\text { blinding }\end{array}$ & $\begin{array}{c}\text { Non- } \\
\text { consecutive } \\
\text { studies or } \\
\text { studies without } \\
\text { consistently } \\
\text { applied } \\
\text { reference } \\
\text { standards }\end{array}$ & $\begin{array}{l}\text { Case-control } \\
\text { study, or poor } \\
\text { or non- } \\
\text { independent } \\
\text { reference } \\
\text { standard }\end{array}$ & $\begin{array}{c}\text { Mechanism- } \\
\text { based } \\
\text { reasoning }\end{array}$ \\
\hline Prognosis & $\begin{array}{c}\text { Systematic } \\
\text { review of } \\
\text { inception } \\
\text { cohort studies }\end{array}$ & $\begin{array}{c}\text { Inception } \\
\text { cohort studies }\end{array}$ & $\begin{array}{c}\text { Cohort study } \\
\text { or control arm } \\
\text { of RCT }\end{array}$ & $\begin{array}{c}\text { Case series, } \\
\text { Case-control } \\
\text { study, or poor } \\
\text { quality } \\
\text { prognostic } \\
\text { cohort study }\end{array}$ & $\begin{array}{l}\text { Expert } \\
\text { Opinion }\end{array}$ \\
\hline Treatment & $\begin{array}{l}\text { SR of RCTs, } \\
\text { SR of nested } \\
\text { case-control } \\
\text { studies, high } \\
\text { quality RCT }\end{array}$ & $\begin{array}{c}\text { RCT (poor } \\
\text { quality) or } \\
\text { observational } \\
\text { study with } \\
\text { dramatic } \\
\text { effect }\end{array}$ & $\begin{array}{c}\text { Non- } \\
\text { randomized } \\
\text { controlled } \\
\text { cohort/follow } \\
\text { up study }\end{array}$ & $\begin{array}{l}\text { Case series, } \\
\text { Case-control } \\
\text { study, or } \\
\text { historically } \\
\text { controlled } \\
\text { studies }\end{array}$ & $\begin{array}{c}\text { Mechanism- } \\
\text { based } \\
\text { reasoning }\end{array}$ \\
\hline Screening & $\begin{array}{c}\text { SR of RCTs, } \\
\text { high quality } \\
\text { RCT }\end{array}$ & $\begin{array}{l}\text { RCT (poor } \\
\text { quality) }\end{array}$ & $\begin{array}{c}\text { Non- } \\
\text { randomized } \\
\text { controlled } \\
\text { cohort/follow } \\
\text { up study }\end{array}$ & $\begin{array}{l}\text { Case series, } \\
\text { Case-control } \\
\text { study, or } \\
\text { historically } \\
\text { controlled } \\
\text { studies }\end{array}$ & $\begin{array}{c}\text { Mechanism- } \\
\text { based } \\
\text { reasoning }\end{array}$ \\
\hline
\end{tabular}

RCT:-randomized controlled trial; SR: systematic review. 